\title{
Cetuximab-induced skin exanthema: Improvement by a reactive skin therapy
}

\author{
CARL C. SCHIMANSKI ${ }^{1}$, MARKUS MOEHLER ${ }^{1}$, TIM ZIMMERMANN ${ }^{1}$, MARKUS A. WÖRNS ${ }^{1}$, \\ ALMA STEINBACH ${ }^{2}$, MICHAEL BAUM ${ }^{2}$ and PETER R. GALLE ${ }^{1}$ \\ ${ }^{1}$ First Department of Internal Medicine, Johannes Gutenberg University of Mainz, \\ 55101 Mainz; ${ }^{2}$ Merck Pharma GmbH, 64271 Darmstadt, Germany
}

Received April 9, 2010; Accepted July 7, 2010

DOI: $10.3892 / \mathrm{mmr} .2010 .336$

\begin{abstract}
More than $80 \%$ of patients treated with cetuximab develop an acneiform follicular skin exanthema. Grade 3 exanthema develops in $9-19 \%$ of these cases, bearing the risk of cetuximab dose-reduction or cessation. We retrospectively analysed a cohort of 20 patients treated with cetuximab and an in-house reactive skin protocol upon development of an exanthema. The reactive skin protocol was built up as follows: grade 1 exanthema: topical cleansing syndet $\left(\right.$ Dermowas $\left.^{\circledR}\right)+$ topical metronidazole cream $\left(\right.$ Rosiced $\left.^{\circledR}\right)$; grade 2 exanthema: grade 1 treatment + oral minocycline $50 \mathrm{mg}$ twice per day; grade 3 exanthema: grade 2 treatment + topical corticoid (Dermatop ${ }^{\circledR}$ ) + topical nadifloxacin $\left(\operatorname{Nadixa}^{\circledR}\right)$. As soon as a grade 3 had improved to a grade less than or equal to 2, the application of the topical corticoid was ceased. During the initial 12 weeks of therapy with cetuximab, all patients developed a skin exanthema $(20 / 20 ; 100 \%)$. Of these, 2 patients $(10 \%)$ developed a grade 3 exanthema, 10 patients $(50 \%)$ experienced a grade 2 and 8 patients (40\%) a grade 1 exanthema. Time to onset ranged from 1 to 4 weeks, with the average time to onset being 2.8 weeks. Applying the reactive skin protocol after the first occurrence of an exanthema, the grade of exanthema was downgraded as follows: no patients $(0 \%)$ had a persisting grade 3 exanthema, while only 2 patients $(10 \%)$ experienced a persisting grade 2 exanthema and 8 patients (40\%) a persisting grade 1 exanthema. In the majority of cases (10 patients; $50 \%$ ), the reactive skin protocol completely controlled the exanthema (grade 0 ). The average time to exanthema reduction by one grade was 9.5 days. No dose reductions of cetuximab were
\end{abstract}

Correspondence to: Dr Carl C. Schimanski, First Department of Internal Medicine, Johannes Gutenberg University of Mainz, Langenbeckstrasse 1, 55101 Mainz, Germany

E-mail: schimanski@1-med.klinik.uni-mainz.de

Abbreviations: EGFR, epithelial growth factor receptor; WHO, world health organization

Key words: skin, rash, exanthema, epithelial growth factor receptor (EGFR), anti-EGFR, cetuximab, therapy, reactive necessary. Cetuximab-induced skin exanthema is effectively managed by applying our reactive protocol. The simple protocol is based on a topical cleansing syndet and topical metronidazole and is to be intensified by the addition of oral minocycline, a topical corticoid and topical nadifloxacine, in cases of high-grade exanthema. More comprehensive results are expected from a prospective study with higher patient numbers that is currently being planned.

\section{Introduction}

Epidermal growth factor receptor (EGFR) inhibitors are used as highly effective therapies for diverse tumour entities, including colorectal, head and neck, non-small cell lung and pancreatic cancers, among other malignancies (1-5). Although EGFR inhibitors, such as cetuximab, are generally well tolerated and do not increase the classical adverse events of chemotherapy, they induce a skin exanthema that occurs in more than $80 \%$ of patients $(1,4)$. While the exanthema has an acneiform appearance, its origin is nonetheless follicular (5). This follicular acneiform exanthema presents itself as maculae, papulae or small pustulae occurring on the face, décolleté or back, and may be accompanied by symptoms, such as burning or itching.

Time to onset is between 2 and 3 weeks after the first application of the EGFR inhibitor. While the majority of patients develop only mild exanthemas (grades 1 or 2), 9-19\% of patients experience severe exanthemas (grades 3 or 4). More severe or persistent exanthemas may occur, particularly with the administration of EGFR antibodies (5). When a severe exanthema occurs, cessation of the EGFR inhibitor is considered, and is undertaken in approximately $10 \%$ of patients (5). Withdrawing EGFR inhibitors from cancer therapy is disquieting for patients, especially as most patients know that the development and intensity of an exanthema is a good surrogate marker for response rates and improved survival (6). Risk factors for the development of severe exanthemas are male gender and age below 70 years (7).

No standardized therapy exists for the treatment of skin toxicities. Usually, strategies established for the therapy of acne are used. Recently, the first randomized trials were conducted analysing the prophylaxis and therapy of cetuximab-induced skin exanthemas $(8,9)$. 
Scope et al reported that oral minocycline prophylaxis resulted in less severe and less symptomatic skin exanthemas during the first 4 weeks (8). However, this effect was only temporary and vanished with continued application. Similarly, Jatoi et al analysed the effect of an oral tetracycline prophylaxis on the development of skin exanthemas of a grade greater than or equal to 2, and found that prophylaxis with tetracycline was effective during the first weeks, but ineffective in a longterm setting (9). These antibiotics are commonly used for the treatment of acne, and the clinical similarity between classical acne and an EGFR inhibitor-induced skin exanthema suggests that this antibiotic may play a role in preventing or treating drug-induced exanthemas $(10,11)$. However, tetra-/doxy-/ and minocycline carry anti-inflammatory effects that may also support exanthema palliation $(11,12)$.

Another randomized Phase II study, termed the STEPPstudy, analysed the effect of a prophylactic vs. a reactive therapy during second-line panitumumab therapy in colorectal cancer (13). The prophylactic therapy consisted of lotion, oral doxycycline, topical hydrocortisone (1\%) and sun block, resulting in a significant reduction in not only skin exanthemas of a grade greater than or equal to 2, but also in the median time to onset of a skin exanthema of a grade greater than or equal to 2 . However, this study has been criticized as all patients randomized in the prophylactic group received topical cortisone. Furthermore, the reactive therapy was not defined, but rather was left open to the physician's discretion.

In view of these data, we retrospectively analysed the patients receiving cetuximab and an in-house reactive skin protocol based on the therapeutic options of classical acne and the treatment recommendations of the cetuximab manufacturer. This was possible since we had established a reactive skin protocol in 2008 that documented skin toxicities according to the National Cancer Institute's Common Terminology Criteria for Adverse Events version 3.0 (NCI CTCAE v3.0) using digital photography.

\section{Materials and methods}

Patients. Lacking standardized guidelines, we had discussed and agreed upon an in-house reactive skin protocol derived from the common acne therapy, which was offered to the patients treated with cetuximab. The reactive skin protocol was first offered to a patient in April 2008. We retrospectively analysed the patients receiving the reactive skin protocol with cetuximab-based chemotherapy as of April 2008.

All adverse events were routinely documented on a weekly basis as per the NCI CTCAE v3.0. The evaluation entailed physical examination, a weekly assessment of patient performance status and weight, and an assessment of adverse events, including gastrointestinal toxicity and exanthema development. Skin toxicities were also documented by digital photography on a weekly basis.

Reactive skin protocol. The reactive skin protocol was established as follows: grade 1 exanthema, topical cleansing syndet $\left(\right.$ Dermowas $\left.^{\circledR}\right)+$ topical metronidazole cream $(7.5 \%)$ on affected skin areas (Rosiced $\left.{ }^{\circledR}\right)$; Grade 2 exanthema, grade 1 treatment + oral minocycline $50 \mathrm{mg}$ twice daily; Grade 3 exanthema, grade 2 treatment + topical corticoid prednicarbat
Reactive skin protocol

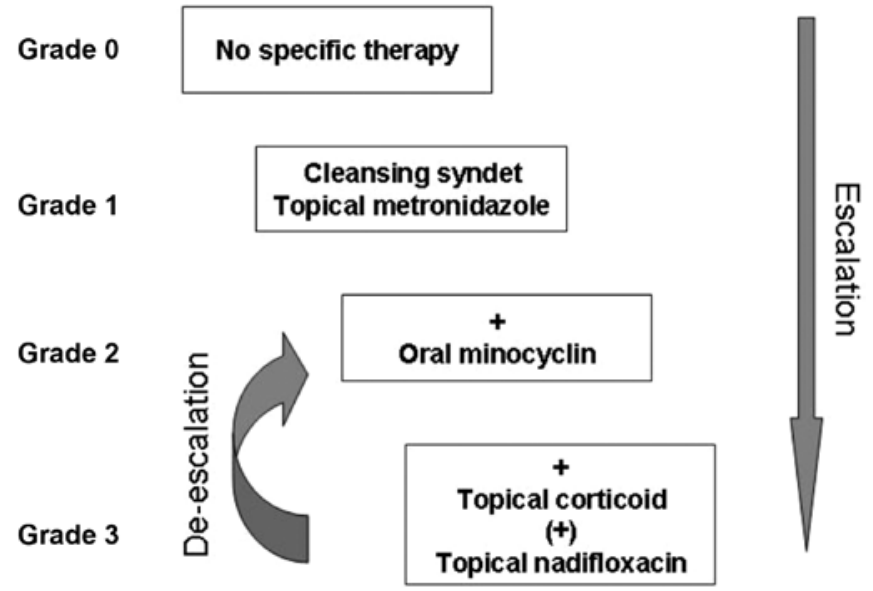

Figure 1. The reactive skin protocol was established as follows: grade 1 exanthema, topical cleansing syndet $\left(\right.$ Dermowas $\left.^{\circledR}\right)+$ topical metronidazole cream $\left(\right.$ Rosiced $\left.^{\circledR}\right)$; grade 2 exanthema, grade 1 treatment + oral minocycline $50 \mathrm{mg}$ twice per day; grade 3 exanthema, grade 2 treatment + topical corticoid $\left(\right.$ Dermatop $\left.{ }^{\circledR}\right)+$ topical nadifloxacin $\left(\right.$ Nadixa $\left.^{\circledR}\right)$. As soon as a grade 3 improved to grade $\leq 2$, the application of the topical corticoid was ceased.

cream $(0.25 \%)$ on affected skin areas (Dermatop $\left.{ }^{\circledR}\right)$ (Fig. 1). Additional topical nadifloxacin cream (1\%) $\left(\mathrm{Nadixa}^{\circledR}\right)$ was applied on affected skin areas only in one severe case of grade 3 exanthema with an increased risk of cutaneous super-infection. As soon as the grade of 3 had improved to a grade $\leq 2$, the application of topical corticoid (and topical nadifloxacin) was ceased.

It was specified that the administration of minocycline was to be ceased in the event of grade 2 nausea and/or vomiting. The latter was characterized by 2-5 episodes of nausea/ vomiting within $24 \mathrm{~h}$.

Statistical analyses. The data were explored in a one-armed retrospective descriptive setting.

\section{Results}

Patients. Between June 2008 and July 2009, 20 patients were treated according to our in-house reactive skin protocol. At the time of retrospective evaluation, all patients had received treatment under this protocol for a minimum of 12 weeks. None of the patients had a history of acne.

The median age of the patients was 65.5 years (range 48-81). Within the entire cohort, $90 \%$ were male and $10 \%$ female. All patients suffered from a stage UICC IV gastrointestinal adenocarcinoma. The patients had received a standard initial cetuximab dose of $400 \mathrm{mg} / \mathrm{qm}$ and thereafter $250 \mathrm{mg} /$ qm weekly combined with either irinotecan or platinum-based chemotherapy. No radiation was applied. Upon restaging after 12 weeks, 9 patients had partial response (45\%), 9 had stable disease (45\%) and 2 had progressive disease (10\%).

Exanthema. During the first 12 weeks of therapy with cetuximab, all patients developed a skin exanthema (20/20; $100 \%)$. Of these, 2 patients (10\%) developed a grade 3 exanthema, while $10(50 \%)$ experienced a grade 2 and $8(40 \%)$ a 


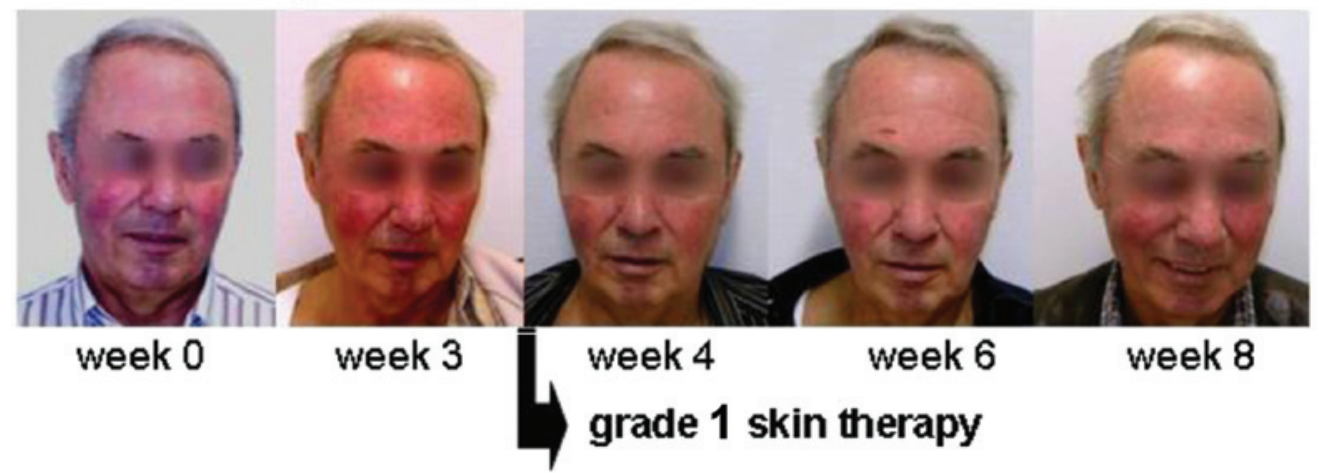

B

\section{Exanthema grade 2}

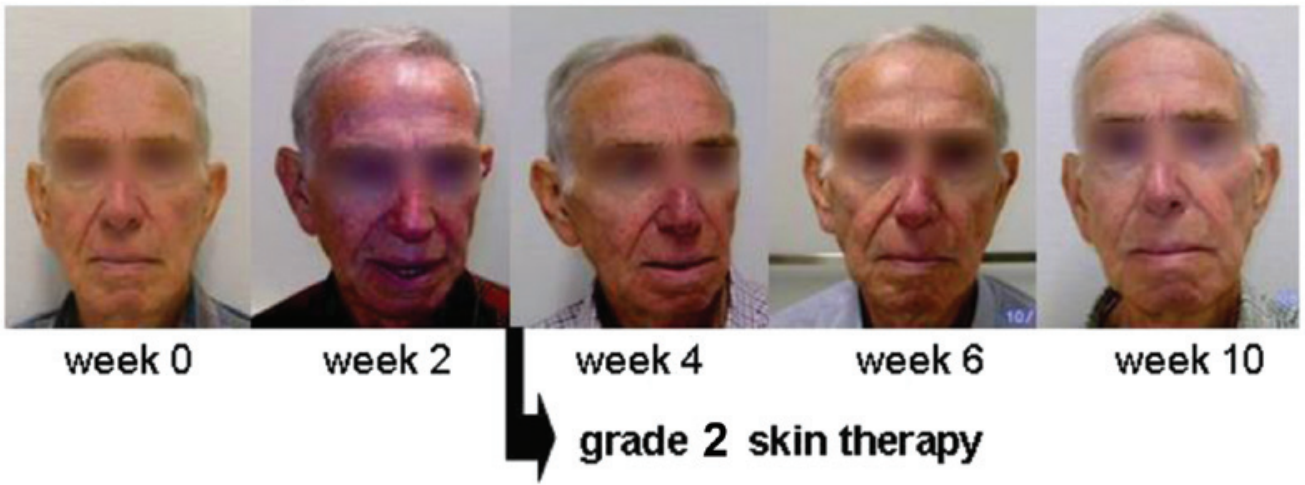

C

Exanthema grade 2-3

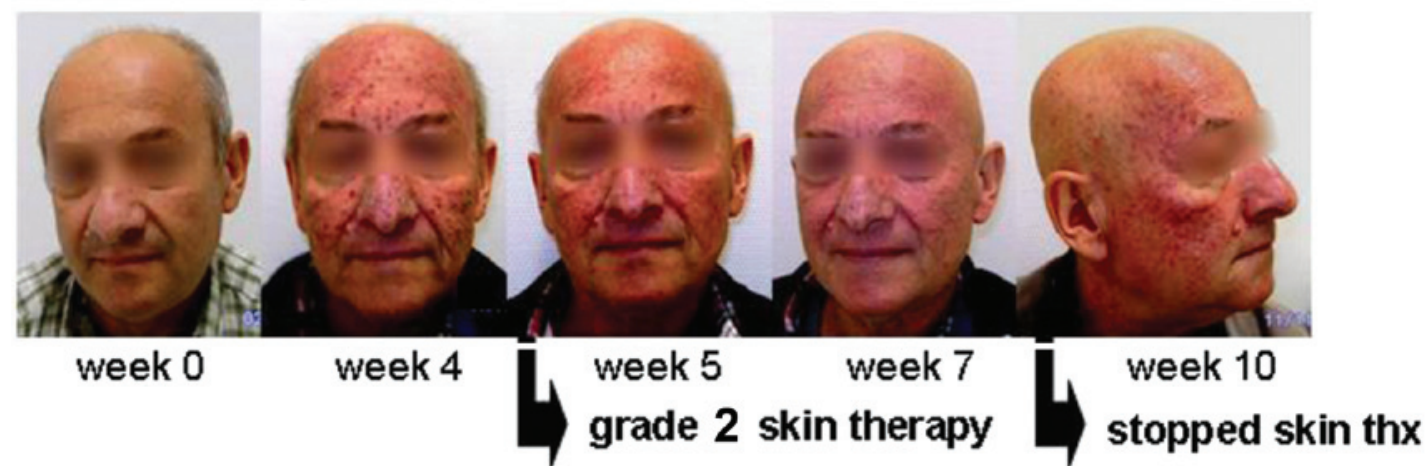

Figure 2. (A) A 65-year-old patient with metastatic colorectal cancer. After 3 weeks of therapy with cetuximab, the patient developed an asymptomatic skin erythema with isolated papulae (grade 1). Upon specific skin therapy with topical cleansing syndet and topical metronidazol cream, the skin improved within 1 week and stayed asymptomatic for the following weeks. Cetuximab treatment was not stopped. (B) A 71-year-old patient with metastatic colorectal cancer. After 2 weeks of therapy with cetuximab, the patient developed asymptomatic erythema and exanthema of the face (except on the upper part of the forehead) with papulae and nose-bound small pustulae. Upon specific therapy with topical cleansing syndet, topical metronidazol cream and oral minocycline, the skin improved within 2 weeks and stayed asymptomatic during the following weeks. Cetuximab treatment was not stopped. (C) A 68-year-old patient with metastatic colorectal cancer. After 4 weeks with cetuximab based therapy, the patient presented with an excoriated symptomatic exanthema grade 2 in early conversion to grade 3, including maculae, papulae and excoriated pustulae. Upon the induction of skin therapy with topical cleansing syndet, topical metronidazol cream and oral minocycline (plus an oral antihistaminic for 1 week), the skin improved rapidly within 1 week and stayed stable as a grade 1 exanthema. Cetuximab treatment was not stopped. However, the patient was incompliant and stopped the reactive skin therapy at week 9 . Within 1 week the skin worsened and he developed a grade 2 exanthema of the cheeks.

grade 1 exanthema (Figs. 2 and 3). Time to onset ranged from 1-4 weeks, with the average time to onset being 2.8 weeks.

The application of the reactive skin protocol upon the first occurrence of an exanthema led to a proactive improvement in the exanthema as follows (Figs. 2 and 3): no patients $(0 \%)$ had a persisting grade 3 exanthema, while only $2(10 \%)$ experi- enced a persisting grade 2 exanthema and $8(40 \%)$ a persisting grade 1 exanthema. In the majority of cases (10 patients; $50 \%$ ), the reactive skin protocol completely controlled the exanthema (grade 0 ). The average time to exanthema reduction by one grade was 9.5 days: 13 patients improved by at least one grade within 7 days, and 7 patients improved by 


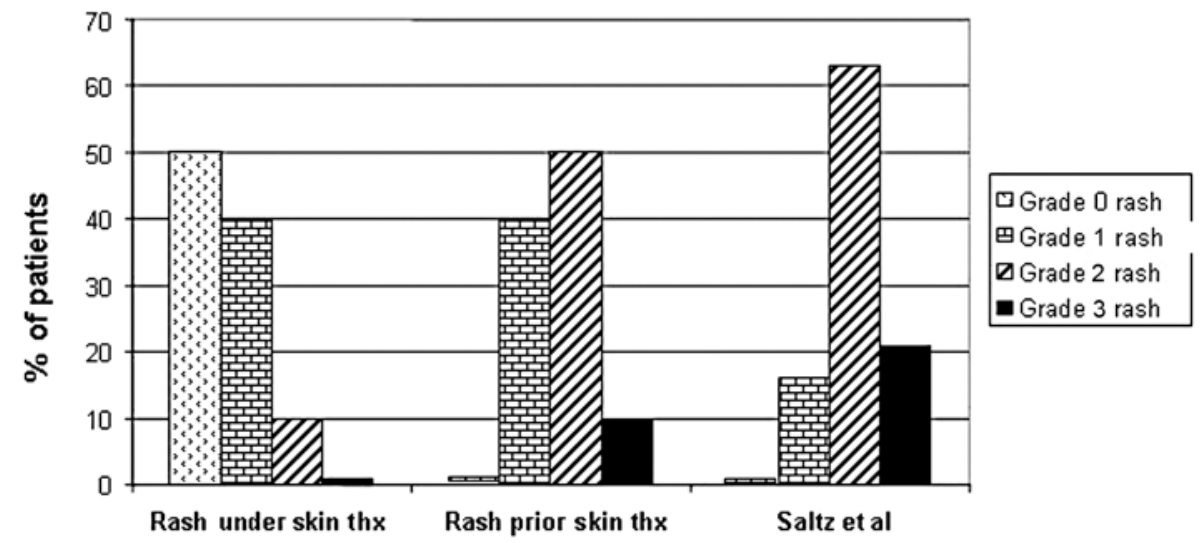

Figure 3. During therapy with cetuximab, all patients developed a skin exanthema (20/20;100\%): 2 patients (10\%) developed a grade 3 exanthema, while 10 (50\%) experienced a grade 2 and $8(40 \%)$ a grade 1 exanthema. Applying the reactive skin protocol after the first occurrence of exanthema, the exanthema intensity was actively suppressed as follows: 0 patients $(0 \%)$ had a persisting grade 3 exanthema, while 2 (10\%) experienced a persisting grade 2 exanthema and $8(40 \%)$ a persisting grade 1 exanthema. In the majority of cases (10 patients; $50 \%)$, the reactive skin protocol completely cleared the exanthema. With regard to exanthema severity, our data suggest that the reactive skin protocol may also have carried a favourable influence on the severity of the exanthema. As compared to the data presented by Saltz et al, the occurrence of grade 3 exanthema among our patients was lower (10 vs. $21 \%)$, as was that of grade 2 (50 vs. $63 \%)$.

at least one grade within 14 days. After initialisation of the skin protocol, additional escalation (from step 1 to step 2 or 3) was necessary for $25 \%$ (5/20 patients) prior to successful exanthema reduction of one or more grades.

With regard to exanthema severity, our data suggest that the reactive skin protocol may also have carried a favourable influence on the severity of the exanthema. As compared to the data presented by Saltz et al, our patients developed fewer grade 3 exanthemas (10 vs. 21\%) and fewer grade 2 exanthemas (50 vs. $63 \%$ ).

Furthermore, 2 patients treated with the reactive skin protocol had previously received cetuximab-based chemotherapy without standardized skin therapy, which had been stopped due to exanthema exacerbation. Upon re-exposure to cetuximab-based chemotherapy flanked with the in-house reactive skin protocol, the 2 patients developed stable exanthemas of grade 1 and 2, respectively. They experienced an initial therapeutic benefit. Upon progression, the cetuximab dose for 1 patient (stable grade 1 exanthema) was doubled, with the exanthema staying stable as grade 1 on the face and grade 2 on the back. Notably, the patient was not fully compliant and did not apply any prescribed topical medications on the back.

No skin-protocol associated adverse events occurred. No patient needed to terminate the in-house reactive skin protocol.

\section{Discussion}

To our knowledge, this is the first study reporting on a reactive skin protocol in a cetuximab-treated patient population. Although half of the patients developed asymptomatic exanthema of a grade greater than or equal to 2 shortly after the initiation of cetuximab therapy, the majority of the patients were stabilized and had either no $(50 \%)$ or only a very light grade $1(40 \%)$ exanthema after the initiation of the reactive skin therapy. In addition, the maximum grades observed were much lower than those reported by Saltz et al. Our results indicate that the exacerbation of cetuximab-induced follicular acneiform exanthema was prevented or reversed by a reactive skin protocol. Usage of this protocol prevented a cetuximab dose-reduction or cessation in patients at risk of this, and adverse effects were not observed.

Only a few studies have reported on the therapeutic options for anti-EGFR skin exanthemas. In most cases, prophylactic approaches were chosen. Prophylaxis with oral antibiotics (tetracycline or minocycline) alone was effective for the early phase of the exanthema during the first 4 weeks of anti-EGFR treatment $(8,9)$. A decrease in the incidence of exanthemas of a grade greater than or equal to 2 in patients assigned to the tetracycline or minocycline group was noted. In addition, tetracycline and minocycyline-treated patients reported other favourable effects on symptoms, including less itching, less burning and stinging and less skin irritation compared to patients treated with a placebo. However, these positive effects wore off after prolonged application. Similarly, topical usage of pimecrolimus, a calcineurin inhibitor, did not result in symptomatic relief or an improvement in the severity of the cetuximab-induced exanthema in a small study presented by Scope et al (14). As anti-EGFR strategies such as cetuximab are part of long-term cancer therapy, isolated usage of oral antibiotics appears to be an insufficient approach.

The STEPP study, presented at this year's ASCO GI, combined oral doxycycline with topical hydrocortisone $(1 \%)$ in a prophylactic setting (13). This approach resulted in a dramatic reduction of more severe exanthema and prolongation of time to exanthema onset of a grade greater than or equal to 2. However, this study has been criticized, as all patients randomized in the prophylaxis group had been exposed to topical hydrocortisone. Furthermore, no standardized reactive procedure for the reactive therapy group had been defined.

In our reactive skin protocol setting, only $10 \%$ of patients, namely those who developed a grade 3 exanthema, actually required topical corticoid for a short period of time (maximum 3 weeks). Thus, it is hypothesized that many patients participating in the STEPP study may have been unnecessarily exposed to hydrocortisone. 
The relevant limitation of our study is its retrospective setting and the number of patients treated. As a consequence, it is difficult to derive any valid clinical recommendations from this report. A prospective study is necessary in order to confirm our observations. Thus, further research on how to prevent and palliate exanthemas that occur from anti-EGFR therapy is required.

\section{References}

1. Shepherd FA, Rodrigues Pereira J, Ciuleanu T, et al: Erlotinib in previously treated non-small-cell lung cancer. N Engl J Med 353 123-132, 2005.

2. Saltz LB, Meropol NJ, Loehrer PJ, Needle MN, Kopit J and Mayer RJ: Phase II trial of cetuximab in patients with refractory colorectal cancer that expresses the epidermal growth factor receptor. J Clin Oncol 22: 1201-1208, 2004.

3. Hochster HS, Haller DG, De Gramont A, et al: Consensus report of the international society of gastrointestinal oncology on therapeutic progress in advanced pancreatic cancer. Cancer 107: 676-685, 2006.

4. Bonner JA, Harari PM, Giralt J, et al: Radiotherapy plus cetuximab for squamous-cell carcinoma of the head and neck. N Engl J Med 354: 567-578, 2006.

5. Busam KJ, Capodieci P, Motzer R, Kiehn T, Phelan D and Halpern AC: Cutaneous side-effects in cancer patients treated with the antiepidermal growth factor receptor antibody C225. Br J Dermatol 144: 1169-1176, 2001.

6. Tang PA, Tsao MS and Moore MJ: A review of erlotinib and its clinical use. Expert Opin Pharmacother 7: 177-193, 2006.
7. Jatoi A, Green EM, Rowland KM Jr, Sargent DJ and Alberts SR: Clinical predictors of severe cetuximab-induced rash: observations from 933 patients enrolled in north central cancer treatment group study N0147. Oncology 77: 120-123, 2009.

8. Scope A, Agero AL, Dusza SW, et al: Randomized double-blind trial of prophylactic oral minocycline and topical tazarotene for cetuximab-associated acne-like eruption. J Clin Oncol 25: 5390-5396, 2007.

9. Jatoi A, Rowland K, Sloan JA, et al: Tetracycline to prevent epidermal growth factor receptor inhibitor-induced skin rashes: results of a placebo-controlled trial from the North Central Cancer Treatment Group (N03CB). Cancer 113: 847-853, 2008.

10. Gammon WR, Meyer C, Lantis S, Shenefelt P, Reizner G and Cripps DJ: Comparative efficacy of oral erythromycin vs. oral tetracycline in the treatment of acne vulgaris. A double-blind study. J Am Acad Dermatol 14: 183-186, 1986.

11. Meynadier J and Alirezai M: Systemic antibiotics for acne. Dermatology 196: 135-139, 1998.

12. Rempe S, Hayden JM, Robbins RA and Hoyt JC: Tetracyclines and pulmonary inflammation. Endocr Metab Immune Disord Drug Targets 7: 232-236, 2007.

13. Lacouture ME, Mitchell EP, Piperdi B, et al: Skin toxicity evaluation protocol with panitumumab (STEPP), a phase II, open-label, randomized trial evaluating the impact of a pre-emptive skin treatment regimen on skin toxicities and quality of life in patients with metastatic colorectal cancer. J Clin Oncol 28: 1351-1357, 2010.

14. Scope A, Lieb JA, Dusza SW, et al: A prospective randomized trial of topical pimecrolimus for cetuximab-associated acnelike eruption. J Am Acad Dermatol 61: 614-620, 2009. 